\title{
Lombalgia durante a gestação: eficácia do tratamento com Reeducação Postural Global (RPG)
}

\author{
Lumbar pain during pregnancy: efficacy of Global Postural Reeducation (GPR) treatment
}

Vinicius Fernandes Barrionuevo Gill ${ }^{1,4}$, Maria José Duarte 0sis ${ }^{2,3}$, Aníbal Faúndes ${ }^{2,3}$

Estudo desenvolvido no

Departamento de Tocoginecologia da Faculdade de Ciências Médicas da UNICAMP - Universidade Estadual de Campinas, como parte da dissertação de mestrado "O efeito da reeducação postural global no tratamento da lombalgia durante a gestação", defendida em 9/2/2009.

1 Aluno de Mestrado do

Departamento de Tocoginecologia da Faculdade de Ciências Médicas da Universidade Estadual de

Campinas (UNICAMP) - Campinas (SP), Brasil.

2 Pesquisador do Centro de Pesquisas em Saúde Reprodutiva de

Campinas (CEMICAMP) - Campinas (SP), Brasil.

${ }^{3}$ Professor Colaborador

Voluntário do Departamento de Tocoginecologia da Faculdade de Ciências Médicas da UNICAMP Campinas (SP), Brasil.

${ }^{4}$ Fisioterapeuta, Movimento - Centro de Estudos e Terapia - Campinas (SP), Brasil.

ENDEREÇO PARA CORRESPONDÊNCIA: Vinicius Fernandes Barrionuevo Gil - Rua Dr. Barbosa de Andrade, 684 - Castelo - CEP: 13.070-158 - Campinas (SP), Brasil - E-mail.: viniciusfbgil@uol.com.br

\section{APRESENTAÇ̃̃}

maio 2010

ACEITO PARA PUBLICAÇÃO nov. 2010

FONTE DE FINANCIAMENTO: Nenhuma

CONFLITO DE INTERESSE: nada a declarar
RESUM0: Realizou-se um estudo clínico comparativo com o objetivo de avaliar o efeito da Reeducação Postural Global (RPG) no tratamento da lombalgia durante a gravidez e sua relação com limitações funcionais das gestantes. Os sujeitos foram 34 gestantes nulíparas atendidas em três centros de saúde e um hospital privado de Campinas, com idade gestacional entre 20 e 25 semanas e queixa de dor lombar. Dezessete gestantes foram submetidas a sessões de RPG durante oito semanas consecutivas, e as demais seguiram orientações de rotina para controle da dor lombar. Em cada sessão de RPG avaliou-se a intensidade da dor percebida pela mulher, antes e após os procedimentos. O grupo controle foi avaliado quanto à intensidade da dor na admissão, às quatro e oito semanas do estudo. Aplicou-se o questionário Roland-Morris para avaliar limitações funcionais, ao início e final do acompanhamento. O grupo de RPG apresentou decréscimo estatisticamente significativo na intensidade da dor antes e depois das sessões. Ao longo do estudo, as mulheres submetidas a RPG tiveram medianas de intensidade da dor e médias do escore de limitações funcionais significativamente menores que as do grupo controle. Análise de covariância indicou que o tratamento com RPG estava associado à menor intensidade da dor percebida ao final do estudo. Conclui-se que a RPG pode dar importante contribuição no tratamento da dor lombar durante a gestação, reduzindo, ao mesmo tempo, as limitações funcionais.

Descritores: gravidez; dor lombar; fisioterapia; exercícios de alongamento muscular.

ABSTRACT: A clinical comparative study was carried out to evaluate the effectiveness of Global Postural Reeducation (GPR) in low back pain treatment during pregnancy, and its association with pregnant women functional limitations. The subjects were 34 nulliparous women attended in 3 public health units and 1 private hospital in Campinas. All women were at 20-25 weeks gestation and experiencing low back pain. Seventeen women were submitted to 8 GPR weekly consecutive sessions and 17 followed the routine recommendations for treating lumbar pain. In each GPR session the low back pain severity was assessed, before and after procedures. The control group had 3 low back pain intensity and treatment evaluation sessions (at admission, 4 and 8 weeks). Roland Morris questionnaire was applied in order to assess functional limitations in the beginning and at the end of the study. Women in the GPR group had a significant decrease of low back pain severity, before and after each session. Throughout the study, women in the GPR group had significantly lower pain medians and lower functional limitations score means than women in the control group. Covariance analysis pointed out that GPR treatment was associated to lower perceived pain intensity at the end of the follow-up. It is concluded that GPR can be an important contribution to the low back pain treatment during pregnancy and also it would help to reduce the damage caused by functional limitations. This will certainly has a positive influence upon women's quality of life.

KeYwords: pregnancy; low back pain; physiotherapy; muscle stretching exercises. 


\section{INTRODUCÃO}

A estabilidade da gestante, comprometida pelo aumento de carga sobre músculos e ligamentos da coluna vertebral, pode provocar indisposições e desconfortos musculoesqueléticos, com prejuízo da sensação de bem-estar físico ${ }^{1}$.

Nesse contexto, a dor lombar, que é considerada distúrbio comum na população em geral, é também sintoma frequente durante a gestação. Vários estudos mostraram que pelo menos $50 \%$ das mulheres vivenciaram algum tipo de dor na coluna durante a gravidez ${ }^{2}$. Em dois estudos mais recentes, observou-se um aumento nesse percentual: Noren et al. ${ }^{3}$ verificaram que $70 \%$ das grávidas tinham algum tipo de dor lombar e que 20\% dessas mulheres permaneciam com esse sintoma após o parto; Martins e Pinto e Silva ${ }^{4}$ relataram que aproximadamente $80 \%$ das mulheres tinham dores na região lombar e pélvica, sendo que 51\% apresentavam dor que interferia significativamente em suas habilidades físicas e qualidade de vida.

As dores nas costas durante a gestação representam, portanto, queixa relevante, tanto pela alta frequência de mulheres acometidas, quanto pela intensidade da dor e desconforto provocado, além de influenciar de modo negativo a qualidade do sono, disposição física, desempenho no trabalho, vida social, atividades domésticas e lazer ${ }^{5}$. Esse contexto coloca a necessidade de tratamentos adequados para a dor lombar durante a gravidez ${ }^{6}$.

A gestação limita a adoção das condutas diagnósticas e terapêuticas, normalmente utilizadas para a lombalgia fora do período gestacional, como alguns exames radiológicos e o uso de alguns fármacos. Assim, as medidas de alívio viáveis nesse período requerem primeiramente a valorização de ações normalmente relegadas a um segundo plano pelo modelo vigente, como a aquisição de novos hábitos posturais, a adequação dos ambientes de trabalho, e o uso de exercícios terapêuticos específicos ${ }^{1}$.

Vários estudos, realizados em distintos países, têm apontado a eficácia de técnicas fisioterapêuticas para o tratamento da dor lombar na gestação ${ }^{7-17}$. No Brasil, identificou-se apenas uma pesquisa ${ }^{16}$ que comparou o método de stretching global ativo - SGA e a orientação médica para resolver as dores lombares e/ou pélvica posterior durante a gestação. Os autores concluíram que o método de exercícios SGA diminuiu e reduziu a intensidade da dor lombar e pélvica posterior durante a gestação, enquanto a orientação médica não produziu o mesmo efeito.

Outra opção de técnica fisioterapêutica para tratar a dor lombar na gestação é a Reeducação Postural Global (RPG). De acordo com os princípios da RPG, as cadeias musculares são constituídas por músculos gravitacionais que trabalham de forma sinérgica dentro da mesma cadeia. Por exemplo, todos os músculos da cadeia posterior possibilitam a manutenção da posição ortostática contra a gravidade. Essa técnica preconiza a utilização de posturas específicas para o alongamento dos músculos organizados em cadeias musculares, sendo considerado de longa duração (aproximadamente 15 minutos em cada postura). Esse tipo de alongamento pode ser benéfico para as gestantes com dor lombar porque o aumento da lordose lombar é comum nesse período, e a RPG contribui para diminuir e harmonizar as tensões musculares na cadeia mestra posterior, principalmente nos músculos paravertebrais da região lombar ${ }^{18}$. Vale ressaltar que o alongamento global também poderá contribuir para um melhor alinhamento corporal, pois durante a gravidez o aumento do peso corporal provoca mudança do centro de gravidade, interferindo na postura, equilíbrio e locomoção ${ }^{1}$.

Apesar dos possíveis benefícios que a adoção da RPG poderia trazer para as gestantes que sofrem com dor lombar, não se encontram na literatura estudos nesse sentido. O presente estudo teve como objetivo estudar o efeito da RPG ${ }^{19}$ no tratamento da dor lombar durante a gestação, bem como sua relação com as limitações funcionais das gestantes.

\section{METODOLOGIA}

Foi realizado um estudo clínico comparativo, de caráter exploratório. Um grupo de gestantes foi submetido a sessões individuais de RPG e outro grupo de gestantes seguiu a rotina do pré-natal.
O cálculo do tamanho da amostra foi baseado na diferença de médias de dor avaliadas por escala análogo visual, para a comparação da evolução ao longo do tempo, considerando-se a maior diferença e maior variabilidade segundo a fórmula desenvolvida por Friendly ${ }^{20}$ para análise de variância para medidas repetidas. Os valores esperados foram estimados a partir dos resultados de Martins e Pinto e Silva ${ }^{16}$, considerando os valores obtidos na primeira, quarta e última sessão, um nível de significância de 5\% e um poder do teste de $70 \%$ ( $\square=30 \%$ ). O tamanho amostral necessário foi de $\mathrm{n}=17$ em cada um dos grupos.

As gestantes que participaram do estudo foram selecionadas dentre mulheres que faziam acompanhamento pré-natal em três Centros de Saúde e entre outras que assistiam palestras de preparação para o parto em um hospital privado da cidade de Campinas. Foram adotados os seguintes critérios de inclusão: dor lombar, nuliparidade, gestação única de baixo risco, idade gestacional entre 20 e 25 semanas, faixa etária de 18 a 40 anos, ausência de doença clínica ou obstétrica, ausência de patologias pré-existentes da coluna vertebral.

As gestantes dos Centros de Saúde foram previamente identificadas através de revisão das fichas obstétricas do prénatal. Ao fazer o primeiro contato com elas - pessoalmente ou por telefone - o pesquisador aplicou uma lista de verificação para saber se elas cumpriam os critérios de inclusão. As gestantes que faziam curso de preparação em um serviço privado foram contatadas durante as aulas, quando também se aplicou a lista de verificação àquelas que se mostraram interessadas em participar do estudo. Ao final desse processo, foram convidadas a participar do estudo $41 \mathrm{mu}$ Iheres, das quais quatro não aceitaram, e três não compareceram ao primeiro seguimento.

A aleatorização das mulheres nos dois grupos foi feita através da utilização de uma lista de números aleatórios gerada por computador. Para as que foram alocadas no grupo de RPG foram agendadas oito sessões semanais, e para as demais se agendaram dois encontros de seguimento. No momento da admissão ao estudo e ao final, todas as mulheres responderam 
o questionário Roland-Morris (citado por Nusbaun et al. 2001) ${ }^{21}$ para avaliar as limitações funcionais resultantes das dores referidas sobre a coluna. A cada sessão de RPG coletaram-se informações sobre a intensidade da dor (antes e depois dos procedimentos) utilizando-se ficha especialmente preparada para isto, que incluía a escala visual da dor, com valores de 0 a $10^{22}$. A cada segmento do grupo de controle também se verificou a intensidade da dor e a realização de algum tratamento para seu alívio.

No momento da admissão das mulheres ao estudo, a dor lombar era confirmada clinicamente da seguinte maneira: dor referida pela gestante quando realizada flexão do tronco, acompanhada de diminuição da amplitude de movimento da coluna lombar e de músculos eretores espinhais doloridos à palpação. Foi feita a diferenciação com a dor pélvica posterior $^{8}$. As avaliações da dor lombar foram realizadas por profissionais de fisioterapia e enfermeiras obstétricas, previamente treinados pelo pesquisador responsável. Esses profissionais não acompanharam a aleatorização - portanto não sabiam em que grupo cada mulher estava alocada. Ao final da participação de cada gestante no estudo os mesmos profissionais conduziram uma nova avaliação completa da dor lombar.

As sessões de RPG duravam cerca de 40 minutos e as gestantes eram submetidas ao alongamento dos músculos da cadeia posterior. Foram utilizadas duas posturas ativas: fechamento do ângulo coxo-femoral e abdução dos membros superiores; fechamento do ângulo coxofemoral com adução dos membros superiores; ambas em contração isométrica ou isotônica em posição cada vez mais excêntrica, sem permitir compensações (alongamento global) ${ }^{19}$. Essas posturas foram escolhidas porque elas atuam no alongamento da cadeia mestra posterior, que é a mais encurtada durante a gestação. Também a postura em que se fez a abdução dos membros superiores foi escolhida para permitir o alongamento da subcadeia anterointerna do membro superior, uma vez que as gestantes tendem a desenvolver rotação interna dos braços e protrusão de ombros.

Nenhuma das gestantes incluídas no estudo foi descontinuada, pois nenhuma faltou às sessões de RPG ou aos seguimentos do grupo de controle. Nenhuma delas também apresentou qualquer complicação clínica que contraindicasse a continuação dos alongamentos.

A variável independente foi o tratamento com RPG (sim ou não) e as dependentes foram a intensidade da dor lombar e o escore de limitações funcionais. Como variáveis de controle foram consideradas idade materna, grau de escolaridade, o índice de massa corporal (IMC), idade gestacional ao início do estudo, local de seleção, presença de dor lombar ao início do estudo durante a flexão do tronco, movimentação do tronco em círculo ou à palpação da musculatura da região lombar, semana de gestação em que a dor teve início, e o uso de medicação para dor.

As variáveis demográficas foram descritas pela média, desvio padrão e valores mínimos e máximos. Para as comparações entre os grupos, utilizaram-se os testes $t$ de Student, para as variáveis continuas com distribuição normal, e, na ausência de normalidade, usou-se o teste de Mann-Whitney. Os testes exatos de Fisher e $\square^{2}$ de Pearson foram empregados para analisar as variáveis categóricas. A distribuição da intensidade da dor, medida pela EAV, no grupo submetido a RPG, foi comparada ao longo do tempo entre os instantes antes e depois do procedimento em cada sessão, com o teste Wilcoxon pareado ${ }^{23}$; a intensidade da dor no grupo de intervenção foi analisada em três momentos através do teste de Kruskal-Wallis, seguido de MannWhitney para comparações múltiplas.

Utilizou-se análise de covariância (ANCOVA) ${ }^{24}$ para avaliar possíveis diferenças entre os grupos (RPG e controle) quanto à intensidade da dor percebida pelas mulheres e às limitações funcionais. Considerou-se o grupo como fator independente e o escore pré-tratamento como uma co-variável. Para obter-se um melhor ajuste dos dados, os valores pré e pós-tratamento foram transformados com a extração da raiz quadrada. Em um segundo momento realizou-se análise de covariância para a intensidade da dor percebida pelas mulheres ao final do estudo, ajustada pela intensidade da dor inicial (escore), o grupo do qual participou cada mulher, a idade, a escolaridade (até o ensino fundamental/ médio ou superior; até o ensino médio/ superior), o IMC, a idade gestacional na admissão ao estudo, o local de seleção (hospital privado/posto de saúde). Os dados foram analisados com auxílio do programa SAS (versão 9.1.3, SAS Institute Inc., Cary, USA).

O protocolo da pesquisa foi aprovado pelo Comitê de Ética em Pesquisa da Faculdade de Ciências Médicas da Universidade Estadual de Campinas (CEP/FCM/UNICAMP), registro CEP $\mathrm{n}^{\circ}$ $570 / 2006$. Todas as mulheres que aceitaram participar assinaram um Termo de Consentimento Livre e Esclarecido.

\section{RESULTADOS}

Os dois grupos de mulheres foram semelhantes quanto à maioria das características estudadas no momento da admissão ao estudo: no grupo de RPG foram alocadas dez mulheres selecionadas em hospital privado e sete nos centros de saúde; no grupo de controle havia seis mulheres procedentes do hospital privado e 11 dos centros de saúde. A mediana da idade gestacional na admissão ao estudo foi de 22 semanas nos dois grupos, e a de início da dor lombar esteve entre as semanas 16 (controle) e 17 (RPG); a mediana do índice de massa corpórea (IMC) foi de 24 em ambos os grupos. Oito gestantes do grupo de RPG e dez do grupo controle apresentavam dor à flexão do tronco no momento da admissão ao estudo, e dez do grupo de RPG e sete do grupo controle tinham dor à palpação da musculatura da região lombar. Os grupos foram diferentes, porém, quanto ao grau de escolaridade e a idade. A maioria (10) das gestantes do grupo de RPG tinha formação superior, enquanto no grupo de controle a maioria (13) havia completado o ensino médio ( $p=0,0020)$. A média de idade dos grupos foi estatisticamente diferente ( $p=0,0019)$ : 29 anos $(\mathrm{DP}=5,2)$ no grupo de RPG e 23,7 anos $(\mathrm{DP}=3,9)$ no grupo de controle (Dados não apresentados em tabelas).

Quando observada a intensidade da dor no grupo de RPG ao longo das oito sessões, verificou-se um decréscimo estatisticamente significativo da dor em cada sessão ao se compararem os momentos antes e depois do procedimento, exceto 
na última sessão, em que a mediana da dor foi igual a zero nos dois momentos (Figura 1). Por outro lado, observou-se aumento significativo ( $p=0,025)$ na intensidade da dor em cada seguimento do grupo de controle, passando a média de 5,76 ao início do estudo para 6,06 após quatro semanas, e chegando a 7 após oito semanas. Também houve diferença significativa entre as médias quando se comparou especificamente a dor referida no início do estudo com a dor no último seguimento, oito semanas depois da admissão ( $p=0,00129)$ (dados não apresentados em tabelas). Quando comparada a intensidade da dor percebida pelas mulheres em ambos os grupos, observaram-se valores semelhantes das médias ao início do estudo (média de 5,2, desvio padrão de 1,5, no grupo de RPG; média de 5,8 e desvio padrão de 1,2 no grupo de controle). Ao final, porém, verificou-se diferença significativa entre os grupos, com menor intensidade no grupo de RPG (média de 0,9 e desvio padrão de 1,3) e maior no grupo de controle (média de 7,0 e desvio padrão de 1,4). Ao avaliarem-se as limitações funcionais resultantes das dores na região lombar ao início e final do estudo, verificou-se diminuição dessas limitações no grupo de RPG e aumento no grupo de controle: a média do escore passou de $7,1(\mathrm{DP}=5,0)$ para 2,3 (DP=2,9) no grupo de RPG, e de $9,5(\mathrm{DP}=4,5)$ para $13,8(\mathrm{DP}=3,8)$ no grupo de controle (Figura 2).

A análise de covariância entre a intensidade da dor percebida ao final do estudo e outras variáveis apontou o tratamento com RPG como variável

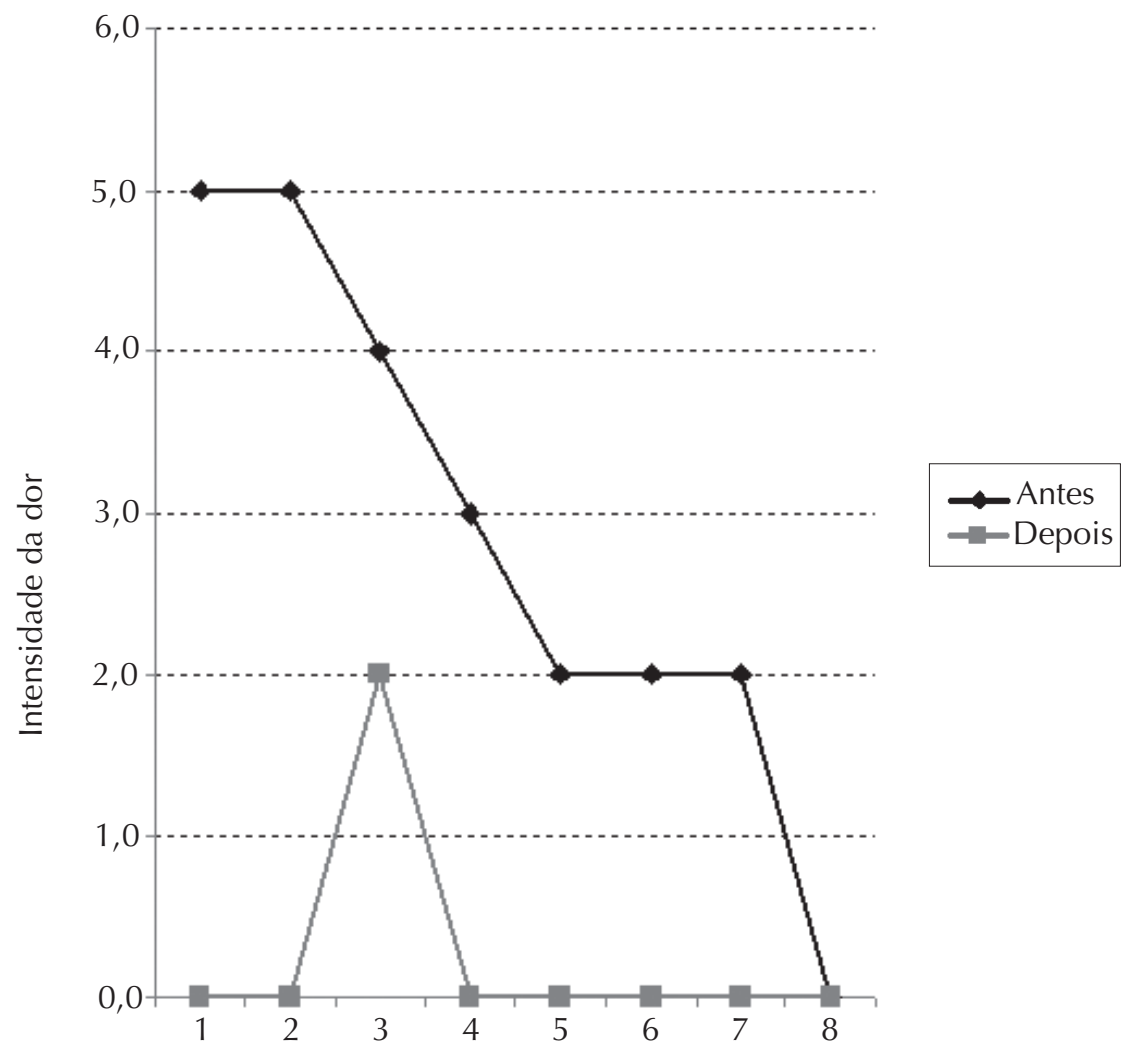

Figura 1. Evolução da intensidade da dor percebida pelas mulheres submetidas a oito sessões de RPG (Teste não paramétrico de Wilcoxon pareado: sessões 1, 2 e $4-p=0,0005$; sessões 3 e $6-p=0,0039$; sessão 5 - p=0,0002; sessão 7 - p=0,0010; sessão 8 - p=0,1250).

fortemente associada ao menor escore de dor ao final do período de seguimento. A menor idade das mulheres e o maior escore de dor ao início do estudo também estiveram associados à menor intensidade da dor percebida pelas mulheres ao final do estudo (Tabela 1).
Quando se comparou a utilização de medicamentos para dor, verificou-se que ela foi mais frequente no grupo de controle do que entre as mulheres que fizeram RPG - $87 \%$ comparados a $12 \%$. Ao longo do seguimento dos grupos não se observou qualquer intercorrência

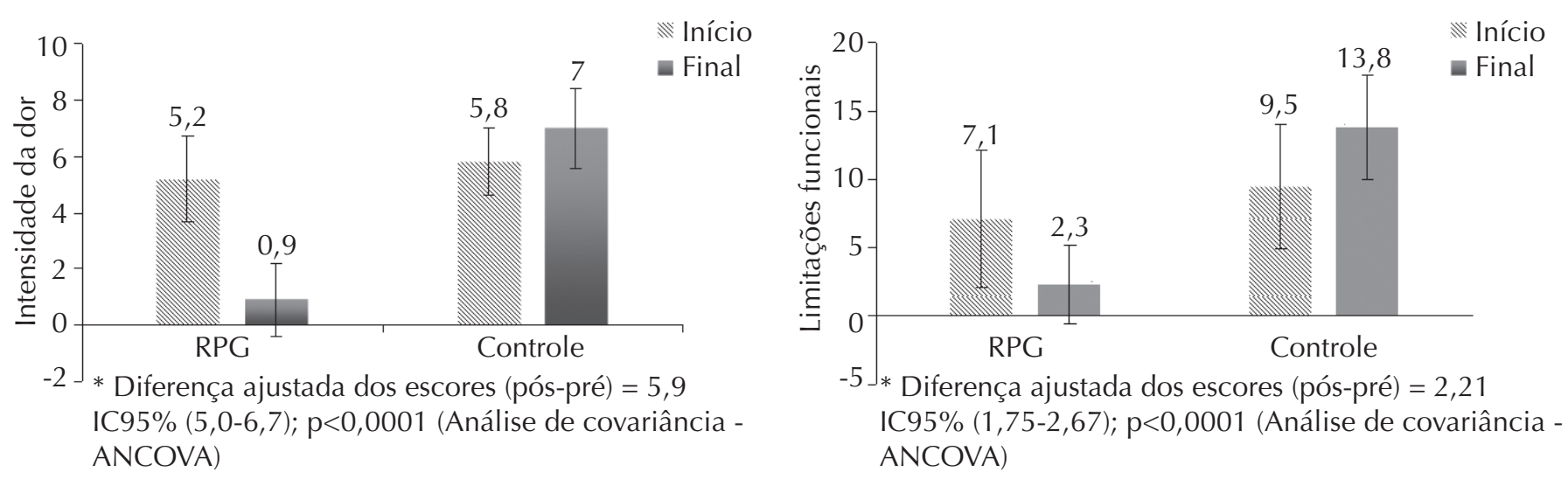

Figura 2. Média e desvio padrão da intensidade da dor percebida pelas mulheres e média e desvio padrão do escore de limitações funcionais, ao início e ao final da intervenção 
Tabela 1. Covariância entre a intensidade da dor percebida ao final do estudo e outras variáveis selecionadas

\begin{tabular}{lccc}
\hline Parâmetros & Coeficientes & Erro padrão & Valor $p$ \\
\hline Intercepto & 0,839 & 3,723 & 0,8237 \\
Grupo RPG & $-6,414$ & 0,518 & $<0,0001$ \\
Idade & 0,154 & 0,06 & 0,0167 \\
Escolaridade: até $1^{\circ}$ grau & 0,699 & 0,891 & 0,44 \\
Escolaridade: até 20 ${ }^{\circ}$ grau & 0,517 & 0,691 & 0,4615 \\
IMC & $-0,055$ & 0,092 & 0,5574 \\
Idade gestacional ao início do estudo & $-0,002$ & 0,103 & 0,981 \\
Local de seleção: hospital privado & 0,461 & 0,404 & 0,2654 \\
Dor ao início do estudo & $-0,445$ & 0,156 & 0,0088 \\
\hline
\end{tabular}

IMC: índice de massa corporal

entre as gestantes (dados não apresentados em tabelas).

\section{DISCUSSÃO}

Nossos resultados indicam que a RPG pode dar uma importante contribuição no tratamento da dor lombar durante a gestação, reduzindo, ao mesmo tempo, as limitações funcionais, o que, certamente, repercute de maneira positiva sobre a qualidade de vida das mulheres. Nossos dados sugerem, ainda, que o tempo de tratamento proposto (oito semanas) foi suficiente para o controle da dor lombar, porém, como a observação se limitou ao período de tratamento, não sabemos por quanto tempo persistiu a remissão da dor.

Como na análise bivariada verificou-se que a maioria das participantes do grupo de RPG tinha maior escolaridade do que as de controle, era possível pensar que isto poderia estar relacionado a melhores condições de vida, menor carga de trabaIho físico e, portanto, melhor controle da dor lombar. Por outro lado, as mulheres do grupo de controle eram mais jovens que as de RPG, o que poderia influenciar a resposta a qualquer tratamento. Ambas as condições poderiam influir indiretamente na dor lombar e estar enviesando os resultados ${ }^{25}$.

Essa possibilidade de que os resultados estivessem enviesados por um vício de seleção das participantes pode ser afastada ao verificar-se, pela análise de covariância, uma forte associação entre a realização de RPG e a diminuição da intensidade da dor percebida pelas mulheres. Os dados, portanto, sugerem fortemente que essa da adoção da RPG como parte das medidas de apoio no pré-natal. Esse tipo de tratamento pode ser vantajoso se comparado a outros por ser uma técnica não invasiva e não apresentar efeitos colaterais imediatos significativos ${ }^{19}, \mathrm{o}$ que é particularmente desejável durante o período gestacional.

No âmbito dos serviços públicos de saúde, a utilização desta intervenção terapêutica implicaria investimentos para contratar ou re-alocar profissionais especializados, porém o montante a ser investido seria pequeno se comparado aos potenciais prejuízos que as limitações funcionais ocasionadas pelas dores lombares podem acarretar na vida das gestantes e no seu desempenho social. Essas limitações estão relacionadas a altos gastos públicos com o afastamento das gestantes de suas atividades cotidianas, inclusive o trabalho remunerado ${ }^{2,6}$. Nos Estados Unidos, por exemplo, os gastos anuais devidos aos afastamentos do trabalho de gestantes com lombalgia chegam a US\$13 bilhões $^{6}$.

Ao mesmo tempo, vale lembrar que prover atendimento que propicie às mulheres maior conforto e menores limitações durante a gestação é condizente com as propostas do Programa de Humanização do Pré-Natal e Nascimento ${ }^{27}$. Além disso, no âmbito do conceito de assistência integral à saúde da mulher, a assistência pré-natal deve também visar, de modo geral, à qualidade de vida das mulheres, e não apenas o diagnóstico e tratamento de complicações médicas durante a gravidez. O Ministério da Saúde ${ }^{27}$ preconiza que os serviços disponham de grupos de apoio no pré-natal, com a participação de equipe multidisciplinar para garantir, ao mesmo tempo, uma abordagem integral e específica das necessidades das mulheres e de seus familiares durante a gravidez. A preparação física da mulher para o parto deve ser um dos alvos desses grupos, o que inclui respiração, relaxamento e exercícios físicos que contribuirão para a melhor resolutividade desse momento, favorecendo o parto vaginal. As atividades desses grupos devem ser complementares às consultas e visar a melhorar a aderência das mulheres às indicações médicas, diminuir suas ansiedades e medos em relação à gravidez, ao parto e ao puerpério. Percebe-se, portanto, que a possível utilização da RPG vem ao encontro dessa 
filosofia de trabalho, e pode ser mais um elemento que contribua para alcançar os objetivos propostos.

\section{CONCLUSÕES}

As gestantes submetidas a RPG apresentaram significativo decréscimo na intensidade da dor lombar ao longo do estudo e em comparação com as gestantes que seguiram as recomendações de rotina no pré-natal para tratar essa dor.

Ao final do estudo, as mulheres submetidas a RPG apresentaram menores limitações funcionais do que as demais.

\section{AGRADECIMENTOS}

Os autores agradecem a Rodrigo Mantelatto Andrade, Claudia Mangetti Pereira da Silva e Regina Grimaldi de Oliveira, pelo auxílio na coleta dos dados e a José Vilton Costa e Sirlei Siani Morais, pela realização da análise estatística dos dados.

\section{REFERÊNCIAS}

1. Polden M, Mantle J. O alívio para incômodo da gravidez. In: Polden M, Mantle J. Fisioterapia em ginecologia e obstetrícia. São Paulo: Santos; 1993.p.133-61.

2. Östgaard HC, Andersson GBJ, Karlsson K. Prevalence of back pain in pregnancy. Spine. 1991;16:549-52.

3. Noren L, Östgaard HC, Johansson G, Ostgaard S. Lumbar back and posterior pelvic pain during pregnancy: a 3-year follow-up. Eur J Spine. 2002;11:267-271.

4. Martins RF, Pinto e Silva JL. Prevalência de dores nas costas na gestação. Rev Assoc Med Bras. 2005;51(3):144-7.

5. Kristiansson P, Svardsudd K, Schoultz BV. Back pain during pregnancy. A prospective study. Spine. 1996;21:702-9.

6. Carr CA. Use of a maternity support binder for relief of pregnancy-related back pain. J Obstet Gynecol Neonatal Nurs. 2003; 32:495-502.

7. Mantle MJ, Holmes J, Currey HLF. Backache in pregnancy II: prophylactic influence of back care classes. Rheumatol Rehabil. 1981; 20:227-32.

8. Östgaard HC, Zetherstrom, G, Roos Hanson, E. - The posterior pelvic pain provocation test in pregnant women. Eur Spine J. 1994;3:258-60.

9. Dumas GA, Reid JG, Wolfe LA, Griffin, McGrath MJ. Exercise, posture, and back pain during pregnancy. Clin Biomech. 1995;10:98-103.

10. Noren L, Östgaard S, Nielsen, TF, Östgaard HC. Reduction of sick leave for lumbar back and posterior pelvic pain in pregnancy. Spine. 1997;22:2157-60.

11. Field T, Reif $M H$, Hart $S$, Theakston H, Schanberg $S$, Kuhn C. Pregnant women benefit from massage therapy. J Psychosom Obstet Gynecol. 1999;20:31-38.

12. Khilstrand M, Stenman B, Nilsson S, Axelsson O. Water gimnastics reduced the intensity of back/low pain in pregnant women. Acta Obstet Gynecol Scand. 1999;78:180-5.
13. Wedenberg K, Moen B, Norling A. A prospective randomized study comparing acupunture with physiotherapy for low-back and pelvic pain in pregnancy. Acta Obstet Gynecol Scand. 2000;79: 331-335.

14. Shim MJ, Lee YS, Oh HE, Kim JS. Effects of a back-painreducing program during pregnancy for Korean women: A non-equivalent control-group pretest-posttest study. Int J Nursing Studies. 2005;1:01-10.

15. Garshasbi A, Zadeh SF. The effect of exercise on the intensity of low back pain in pregnant women. Int J Gynecol Obstet. 2005;88:271-75.

16. Martins RF, Pinto e Silva JL. Tratamento da lombalgia e dor pélvica posterior na gestação por um método de exercícios. Rev Bras Ginecol Obstet. 2005;27(5): 275-82.

17. Granath AB, Helgren MS, Gunnarsson RK. Water aerobics reduces sick leave due to low back pain during pregnancy. J Obstet Gynecol Neonatal Nurs. 2006;35(4):465-71.

18. Fernándes-de-las-Peñas C, Alonso-Blanco C, AlguacilDiego IM, Miangolarra-Page JC. One-year follow-up of two exercise interventions for the management of patients with ankylosing spondylitis. Am J Phys Med Rehabil. 2006;85:559-67.

19. Souchard PE. RPG: Fundamentos da Reeducação Postural Global. 1ª ed. São Paulo: É Realizações; 2005.

20. Friendly M. SAS System for Statistical Graphics. First Edition; Copyright(c) 1995 by SAS Institute Inc., Cary, NC, USA - version 1.2.

21. Nusbaum L, Natour J, Ferraz MB, Goldenberg J. Translation, adaptation and validation of the RolandMorris questionnaire - Brazil Roland-Morris. Braz J Med Biol Res. 2001;34(2)203-210.

22. Olsen S, Nolan M F, Kori S. Pain measurement: an overview of two commonly used methods. Anesthesiol Rev. 1992;6:11-5.

23. Altman DG. Pratical Statistics for Medical Research. London: Chapman \& Hall; 1991. 


\section{Referências (cont.)}

24. Bonate P L. Analysis of Pretest-Posttest Designs. Boca Raton: Chapman \& Hall; 2000.

25. Ferreira $\mathrm{CHJ}$, Nakano MAS. Lombalgia na gestação: etiologia, fatores de risco e prevenção. Femina. 2000;28:435-8.
26. Rosário JLR, Marques AP,Maluf SA. Aspectos Clínicos do alongamento: uma revisão da literatura. Rev Bras Fisioter. 2004;8:83-8.

27. Ministério da Saúde. Secretaria de Políticas de Saúde. Pré-natal, parto e puerpério. Assistência humanizada à mulher. Brasília; 2001. 